\title{
A case report of an extremely rare type of cardiac tumor: Primary Cardiac Angiofibroma
}

\author{
Aisa Talebi ${ }^{1}$, Sam Zeraatian Nejad Davani ${ }^{2}$, Ali Saberi Shahrbabaki ${ }^{1}$, Milad Gholizadeh \\ Mesgarha $^{2}$, Arash Pour Mohammad ${ }^{2}$, and Ali Zare-Mirzaie ${ }^{2}$ \\ ${ }^{1}$ Shahid Beheshti University of Medical Sciences School of Medicine \\ ${ }^{2}$ Iran University of Medical Sciences
}

December 16, 2021

\begin{abstract}
Cardiac angiofibroma is a very rare diagnosis when a patient develops an intracardiac mass. It is a primary benign cardiac tumor with a scarcity of information in the literature. This case report illustrates a 26-year-old man with a complaint of chronic chest tightness who was firstly diagnosed with right ventricle tumor by echocardiography then underwent cardiac MRI which confirmed the presence of a highly-vascular tumor with radiologically benign behavior. Then his tumor was excised, his postoperative course was uncomplicated and he was well within almost 2 months after discharge. Ultimately the histopathologic findings demonstrated vascular and stromal tissue in favor of angiofibroma and excluded the other diagnoses with IHC and trichrome staining. Angiofibroma is a benign, highly vascular tumor, mostly discovered in the nasopharynx. When it is found in the heart, CMR and pathology are pivotal to rule in its diagnosis. It is isointense in T1 weighted and hyperintense in T2 weighted sequences with intense enhancement following contrast injection. Its pathology contains an admixture of vasculatures with CD31 positive immunoreactivity for endothelial cells and fibrotic tissue with bluish coloration in trichrome staining. Eventually, its treatment includes merely surgical excision given its benign nature.
\end{abstract}

A case report of an extremely rare type of cardiac tumor:

Primary Cardiac Angiofibroma

Authors: Aisa Talebi ${ }^{1}$, Sam Zeraatian Nejad Davani ${ }^{2}$, Ali Saberi Shahrbabaki ${ }^{1}$, Milad Gholizadeh Mesgarha $^{3}$, Arash Pour Mohammad ${ }^{3}$, Ali Zare-Mirzaie ${ }^{4}$

\section{Affiliations:}

1. MD, Faculty of Medicine, Shahid Beheshti University of Medical Sciences, Tehran, Iran.

2. MD, Department of cardiovascular surgery, Rasool akram hospital, Iran University of Medical Sciences (IUMS), Tehran, Iran

3. MD, Faculty of Medicine, Iran University of Medical Sciences (IUMS), Tehran, Iran.

4. MD, Department of Pathology, Iran University of Medical Sciences (IUMS), Tehran, Iran.

\section{Corresponding Author Contact Information}

- Name: Milad Gholizadeh Mesgarha

- Address: Rasool-E-Akram Medical Center, Niayesh St., Sattarkhan Ave., Tehran, Iran

- Telephone number: $(+98) 9128142523$

- Postal code: 1445613131 
- FAX number: $+982188602208,+982188915410$

\title{
Authors information:
}

\section{Aisa Talebi, MD}

Faculty of Medicine, Shahid Beheshti University of Medical Sciences, Tehran, Iran

Email : draisatalebi@gmail.com

ORCID : 0000-0001-6388-7639

\section{Sam Zeraatian Nejad Davani, MD}

Cardiovascular surgeon, Department of cardiovascular surgery, Rasool akram hospital, Iran University of Medical Sciences (IUMS), Tehran, Iran

Email: Samzeraatian@yahoo.com

\section{Ali Saberi Shahrbabaki, MD}

Faculty of Medicine, Shahid Beheshti University of Medical Sciences, Tehran, Iran

Email : drsaberiali@gmail.com

ORCID : 0000-0003-0578-8004

\section{Milad Gholizadeh Mesgarha, MD}

Faculty of Medicine, Iran University of Medical Sciences (IUMS), Tehran, Iran.

Email: drmiladgholizadeh@gmail.com

ORCID: 0000-0002-5101-6112

\section{Arash Pour Mohammad, MD}

MD, Faculty of Medicine, Iran University of Medical Sciences (IUMS), Tehran, Iran.

Email: arashpd@gmail.com

ORCID: 0000-0003-3064-4299

\section{Ali Zare-Mirzaie}

Associate Professor of Pathology Department of Pathology, Iran University of Medical Sciences

Email : zaremir@gmail.com

\begin{abstract}
Cardiac angiofibroma is a very rare diagnosis when a patient develops an intracardiac mass. It is a primary benign cardiac tumor with a scarcity of information in the literature.

This case report illustrates a 26-year-old man with a complaint of chronic chest tightness who was firstly diagnosed with right ventricle tumor by echocardiography then underwent cardiac MRI which confirmed the presence of a highly-vascular tumor with radiologically benign behavior. Then his tumor was excised, his postoperative course was uncomplicated and he was well within almost 2 months after discharge. Ultimately the histopathologic findings demonstrated vascular and stromal tissue in favor of angiofibroma and excluded the other diagnoses with IHC and trichrome staining.

Angiofibroma is a benign, highly vascular tumor, mostly discovered in the nasopharynx. When it is found in the heart, CMR and pathology are pivotal to rule in its diagnosis. It is isointense in T1 weighted and hyperintense in $\mathrm{T} 2$ weighted sequences with intense enhancement following contrast injection. Its pathology contains an admixture of vasculatures with CD31 positive immunoreactivity for endothelial cells and fibrotic
\end{abstract}


tissue with bluish coloration in trichrome staining. Eventually, its treatment includes merely surgical excision given its benign nature.

Keywords: Cardiac tumor; Cardiac mass; Angiofibroma; benign tumor; Cardiac magnetic resonance imaging; Histopathology; Median sternotomy; Minimally invasive cardiac surgery

\section{Introduction}

Cardiac tumors can be classified as primary versus metastatic tumors. The primary cardiac tumors (PCTs) are rare tumors that are further categorized as either benign or malignant (1). Their overall prevalence is between $0.0017 \%$ and $0.028 \%$ with predominantly benign tumors $(75-80 \%)(1,2)$. Among the primary benign cardiac tumors, myxoma is the most common one followed by lipoma, fibroelastoma, rhabdomyoma, fibroma, hemangioma, teratoma, cystic tumor, and paraganglioma respectively (1). Herein we report a case of a young adult man with primary cardiac angiofibroma which is also a primary benign cardiac mass but it is extremely rare insofar as being limited to a few case reports in the medical literature $(3,4)$. Thereafter, we discuss its diagnostic features including cardiac MRI and histopathologic findings with the aim of increasing awareness of this infrequent entity.

\section{Case presentation}

A 26-year-old-man presented to the outpatient clinic of cardiac surgery with a complaint of left hemithorax chest tightness and a cardiac mass revealed by echocardiography. The onset of his symptoms was one year before presentation when he developed slowly progressive left-hemithorax chest heaviness and mild dyspnea. He did not report palpitation, syncopal episodes, diaphoresis, lower limbs edema, or severe dyspnea. He did not have a significant medical history or family history of cardiovascular or pulmonary diseases. In his diagnostic investigations prior to his presentation, electrocardiography (ECG) showed $\mathrm{T}$ wave inversion in precordial leads of V3 to V6. To evaluate myocardial ischemia, a myocardial perfusion scan was done which indicated moderate ischemia of the apex, septum, and anterior wall but preserved left ventricular ejection fraction and proper wall motion and thickness. Transthoracic echocardiography indicated immobile nonhomogeneous lobulated mass in the right ventricle (RV) apex (Figure 1). According to the aforementioned findings, he was referred to our cardiac surgery unit. Upon physical examinations, he had normal heart sounds without murmurs and other organs exams were unremarkable. To further characterize the patient's cardiac tumor, cardiovascular magnetic resonance imaging (CMR) was advocated (Figure 2). It showed a round intracavitary mass in the RV apex with well-defined borders and attachment which was isointense in T1 weighted-sequence and hyperintense in T2 weighted and STIR sequences. Additionally, after administration of gadolinium and in the first-pass perfusion imaging, the mass showed avid homogenous enhancement and on late post-contrast images, the mass exhibited intense contrast uptake and gadolinium enhancement. Chest CT scan and laboratory studies comprising complete blood count, coagulation studies, metabolic and electrolytes panels were unremarkable. Given these workup results particularly CMR which was in favor of a highly vascular mass, the patient was diagnosed clinically and radiologically with a benign cardiac tumor, probably hemangioma. The patient underwent tumor resection (Figure 3) and was discharged 3 days postoperatively without any complication. Histopathologic examination was performed which was not in favor of hemangioma (Figure 4). Despite revealing vascular tissue in hematoxylin and eosin staining, interspersing fibrotic tissue was prominent which made the diagnosis of angiofibroma more likely. To exclude other possible diagnoses like hamartoma, immunohistochemical (IHC) staining with CD31 and desmin besides trichrome staining was done which confirmed the diagnosis of cardiac angiofibroma. Eventually, the patient's symptoms markedly abated soon after surgery and in his follow-up, he was doing well with no recurrence of symptoms after 2 months of discharge.

\section{Discussion}

Angiofibroma is a benign, highly vascular tumor that is most commonly found within the nasopharyngeal region in the male adolescent population $(5,6)$. According to the literature, angiofibroma has a heterogeneous appearance with intermediate in T1 weighted and intermediate to high signal intensity in T2 weighted MRI as was the case about our patient CMR even though in another case of cardiac angiofibroma reported by 
Kim et al. hyperintensity was observed in both T1 and T2 weighted sequences (3). Furthermore, considering the profound vascular component of angiofibroma, MRI shows intense enhancement following gadolinium contrast administration which also came true in our patient CMR; though we did not consider angiofibroma firstly as a diagnosis, because given the prevalence, hemangioma as an intensely vascular tumor is more probable than angiofibroma $(1,6,7)$. One of the differences disclosed in the literature between hemangioma and angiofibroma CMR is that hemangioma exhibits faster enhancement than angiofibroma during the infusion of the contrast agent (3). Therefore, in general, we should add angiofibroma to our differential diagnoses besides hemangioma when we confront a benign vascular cardiac tumor based on CMR.

In addition to CMR which is the most comprehensive imaging modality to identify and evaluate cardiac masses, histopathologic evaluation is crucial to confirm the diagnosis of cardiac angiofibroma (1,3). Angiofibroma pathology consists of 2 main components: vascular and stromal tissue; a rich network of variably sized, irregularly shaped vessels surrounded by a low to moderately cellular stroma with thick and thin fibrils of collagen must be observed in microscopic assessment $(4,5)$. Moreover, to confirm the presence of vessels and fibrosis and essentially rule out other diagnoses like hamartoma ( which contains smooth muscles ), IHC and trichrome staining are required. CD31 IHC staining is utilized to indicate the presence of endothelial cells, Desmin IHC is employed to show the existence of muscles and trichrome staining makes fibrotic tissue blue while it makes muscles appear reddish (3).

In view of the management of a cardiac tumor, an interdisciplinary team comprising a cardiac surgeon, cardiologist, and oncologist is required (1). Nonetheless, there is a paucity of information about the treatment of cardiac angiofibroma due to its rarity, considering the benign cardiac tumors, surgical treatment results in a favorable prognosis with a very low recurrence rate (8); consequently, we can presume a similar scenario for cardiac angiofibroma which has benign behavior but a long-term follow-up warrants consideration. Finally in respect of surgical approach, besides traditional median sternotomy (MS) which is a gold standard treatment for PCTs and has great early and late clinical outcomes, an emerging approach is minimally invasive (MI) surgery. It has shown to have appreciable early and late clinical outcomes with a low recurrence rate based on a recent systematic review (9). Although the MS approach was used for our patient, it is recommended to consider the MI technique in the excision of benign cardiac tumors including cardiac angiofibroma.

\section{Figure Legends:}

Figure-1: Transthoracic echocardiography shows an immobile non homogeneous lobulated mass with a size of $37 \times 28 \mathrm{~mm}^{2}$ in the right ventricle (RV) apex.

Figure-2: Cardiac MRI depicts a round ( $35 \times 35$ x 31 cubic millimeters ) intracavitary mass in the RV apex with well-defined borders and attachment versus infiltration of the RV side of the apical septal segment and apical wall of the right ventricle. There is no evidence of pericardial reaction or extracardiac invasion.

A: On steady-state free precession (SSFP) cine images - coronal view, the mass appears slightly and nonhomogeneously hyperintense relative to normal myocardium (arrowheads).

B: On fast spin-echo T1 weighted-sequence - sagittal view, the mass appeared homogenous isointense relative to normal myocardium (arrowheads) as it was in T1 weighted-sequence with fat suppression (not shown here).

C: On T2 weighted-sequence - axial view, the mass appeared homogeneous hyperintense relative to normal myocardium (arrowheads) .

D: On STIR sequences - sagittal view, the mass appeared homogeneous hyperintense relative to normal myocardium (arrowheads).

Figure-3: A: An extensive tumor with the origin of the right ventricle is evident (arrows).

B: Gross pathology of the resected tumor denotes a whitish brown solid mass.

Figure-4: Microscopic pathology demonstrates admixture of vasculatures with irregular, varying size lumina (arrowheads) interspersed by eosinophilic fibroblasts and collagen depositions (arrows) in favor of angiofibro- 
ma (H \& E x 100) (A); Vascular spaces shows positive CD31 immunoreaction (arrows) which supports the endothelium presence (CD31 x 400) (B); Desmin immunohistochemical (IHC) staining indicates reactivity only in the smooths muscles around the vessels but stroma did not show any reactivity (Desmin x 100) (C); On trichrome staining, the most of the stroma depicts bluish coloration which is further supportive of fibrotic tissue (D) whereas, in picture D window, the myocardial muscle took red-brown color favored the muscle existence (absent in tumor pathology section) (trichrome x 100). Desmin IHC and trichrome staining findings confirmed the diagnosis of Angiofibroma.

\section{Acknowledgements}

None.

\section{Funding}

No funding was received for writing this article

\section{Patient Consent}

No identifiable information was disclosed in writing this article, however written consent was obtained from the patient.

\section{Conflict of Interest}

The authors declare no conflict of interest.

\section{References}

1. Poterucha TJ, Kochav J, O'Connor DS, Rosner GF. Cardiac Tumors: Clinical Presentation, Diagnosis, and Management. Curr Treat Options Oncol. 2019 Jun;20(8):66.

2. Gupta R, Meghrajani V, Desai R, Gupta N. Primary Malignant Cardiac Tumors: A Rare Disease With an Adventurous Journey. J Am Heart Assoc [Internet]. 2020 May 18;9(10):e016032. Available from: https://doi.org/10.1161/JAHA.120.016032

3. Kim YJ, Kim YJ, Kim SH, Youn Y-N, Park S. Primary cardiac angiofibroma. Korean Circ J. 2013 Sep;43(9):636-9.

4. Issa OM, Reyna J, Santana O, DeBeer S, LaPietra A, Lamelas J. Cardiac Angiofibroma: A Rare Primary Cardiac Tumor. J Card Surg [Internet]. 2013 Jul 1;28(4):404-5. Available from: https://doi.org/10.1111/jocs. 12081

5. Stelow EB, Wenig BM. Update From The 4th Edition of the World Health Organization Classification of Head and Neck Tumours: Nasopharynx. Head Neck Pathol. 2017 Mar;11(1):16-22.

6. Tork CA, Simpson DL. Nasopharyngeal Angiofibroma. StatPearls. 2021.

7. Das A, Bhalla AS, Sharma R, Kumar A, Thakar A, Vishnubhatla SM, et al. Can Diffusion Weighted Imaging Aid in Differentiating Benign from Malignant Sinonasal Masses?: A Useful Adjunct. Polish J Radiol. 2017;82:345-55.

8. Rahouma M, Arisha MJ, Elmously A, El-Sayed Ahmed MM, Spadaccio C, Mehta K, et al. Cardiac tumors prevalence and mortality: A systematic review and meta-analysis. Int J Surg. 2020 Apr;76:178-89.

9. Moscarelli M, Rahouma M, Nasso G, di Bari N, Speziale G, Bartolomucci F, et al. Minimally invasive approaches to primary cardiac tumors: A systematic review and meta-analysis. J Card Surg. 2021 Feb;36(2):483-92. 


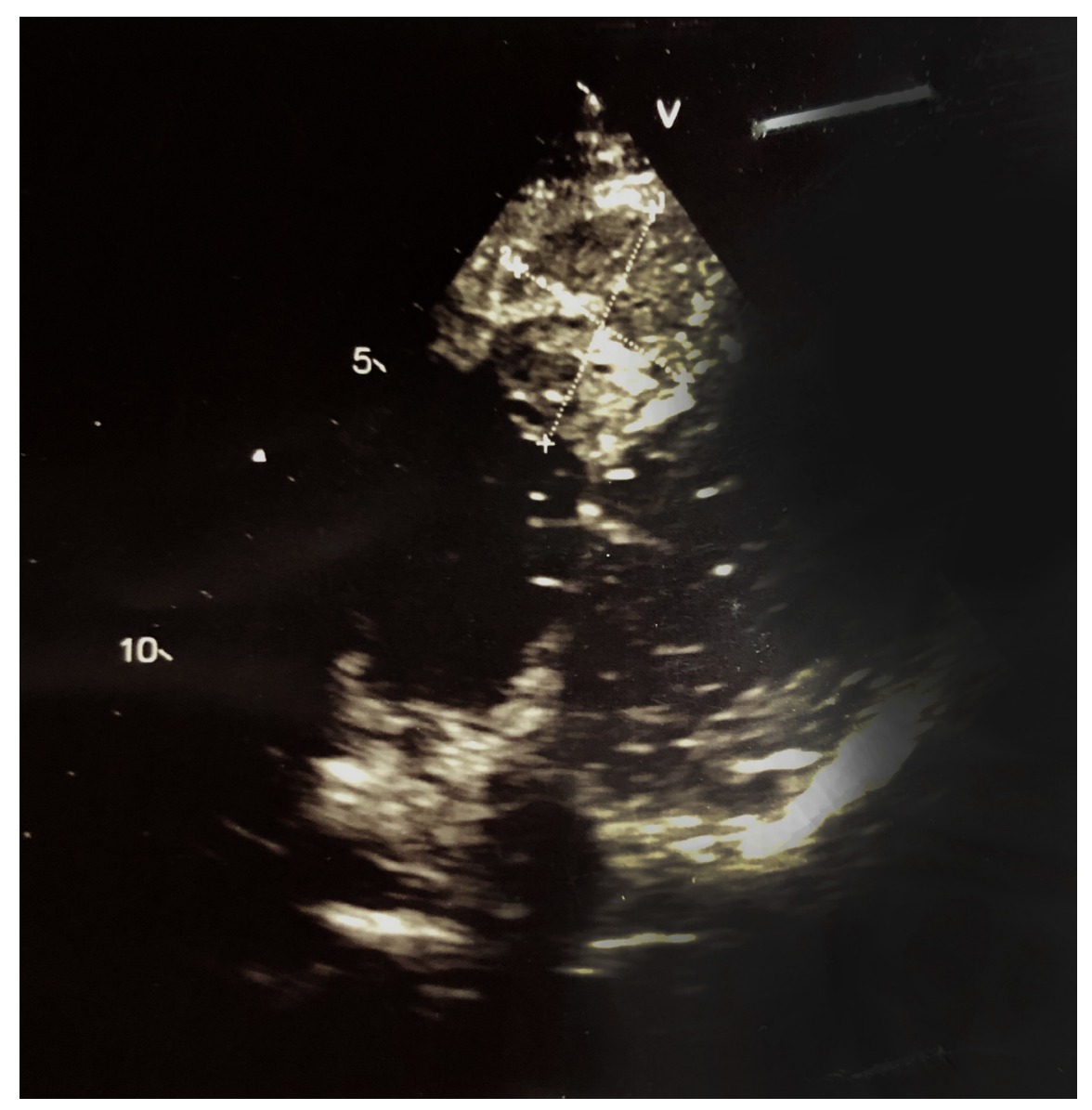



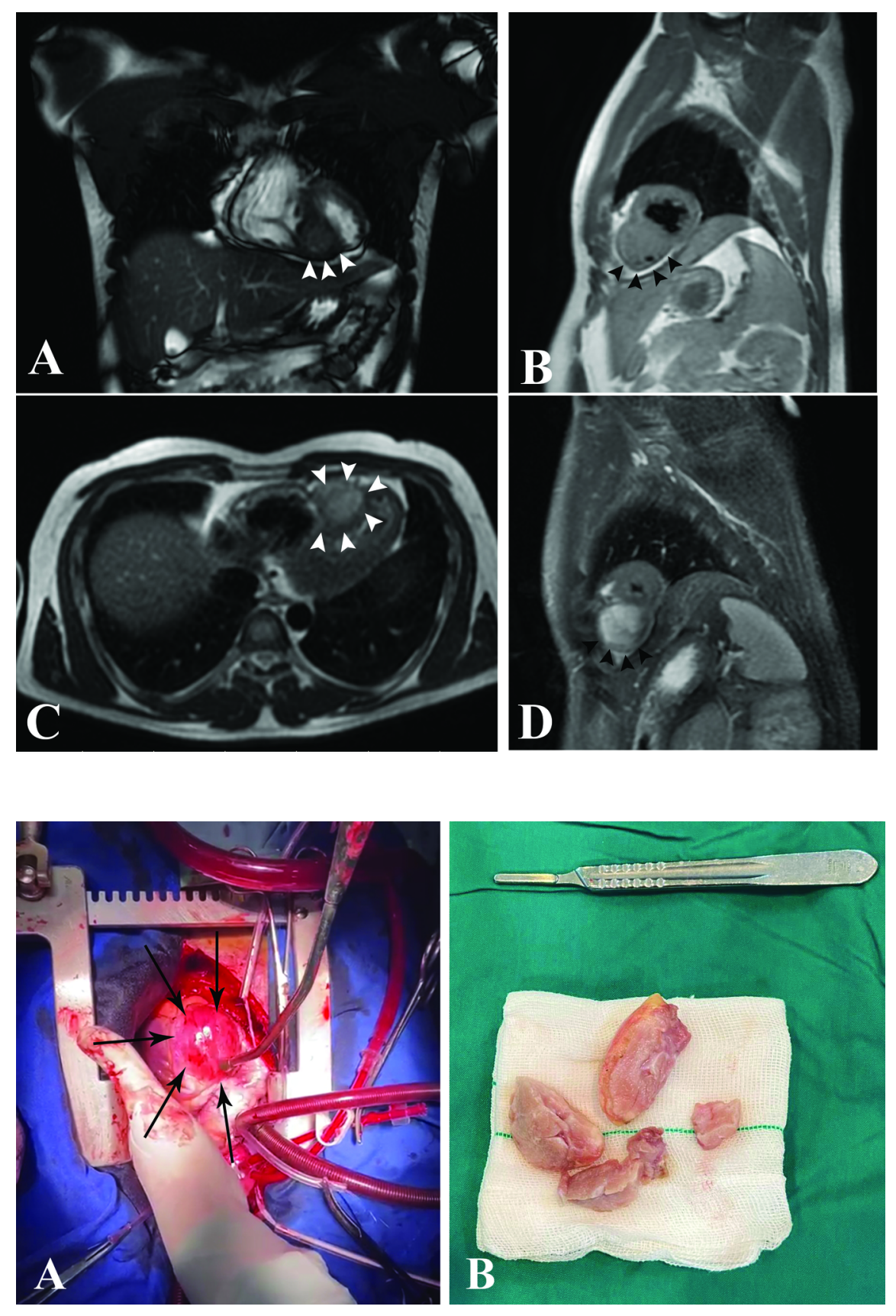


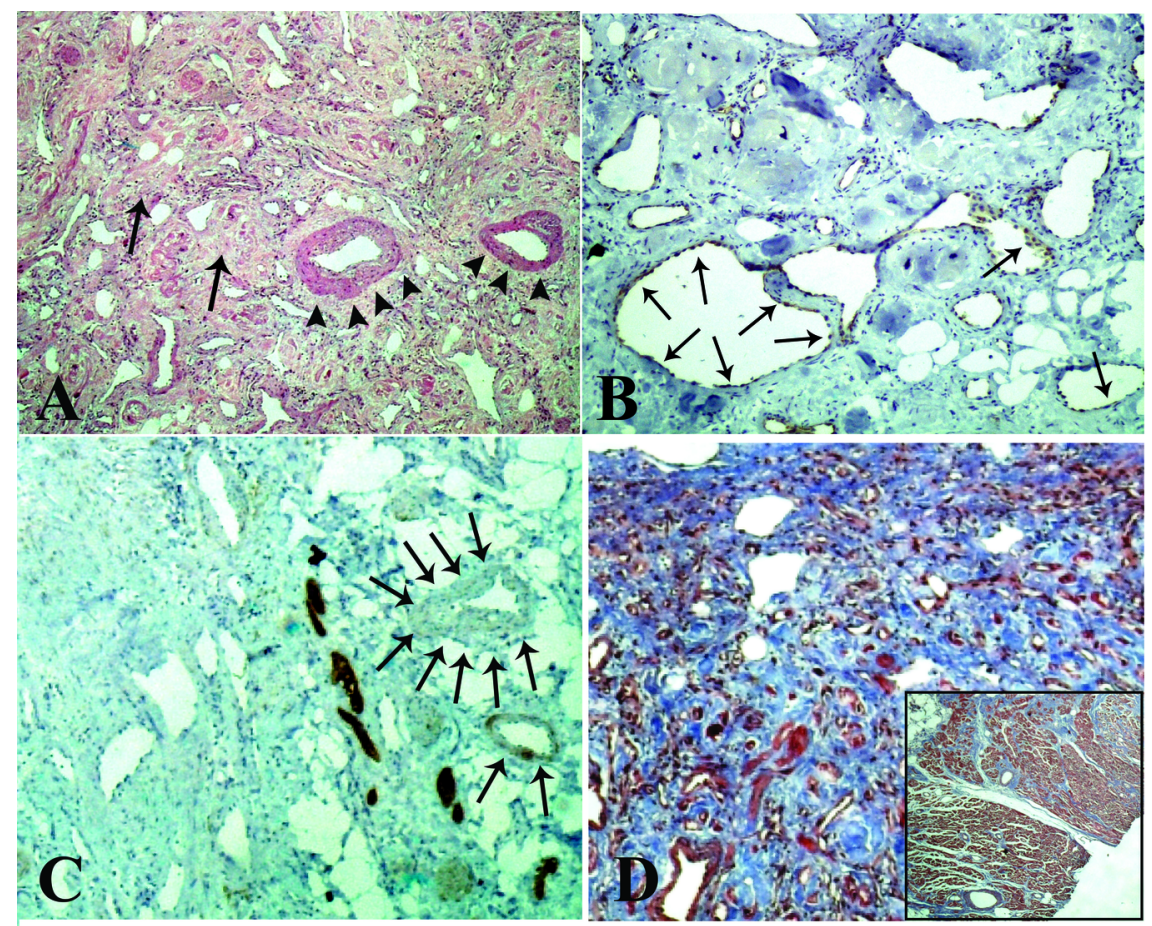

